\title{
Fibromyalgia: practical considerations for oral health care providers
}

Younghoon Jeon

Department of Anesthesiology and Pain Medicine, School of Dentistry, Kyungpook National University, Daegu, Republic of Korea

Fibromyalgia is a syndrome characterized by chronic pain in the skeletal system accompanied by stiffness, sleep disturbance, fatigue, and psychiatric problems, such as anxiety and depression. Fibromyalgia commonly affects orofacial health, presenting with a variety of oral manifestations, including temporomandibular disorder, xerostomia, glossodynia, and dysgeusia. Therefore, oral healthcare providers need to be aware of this clinical entity to effectively manage oral symptoms and provide proper oral self-care modification and education on the nature of fibromyalgia. This review focuses on the epidemiology, pathophysiology, clinical manifestation, diagnosis, orofacial concerns, and treatment of fibromyalgia.

Keywords: Dysgeusia; Fibromyalgia; Glossodynia; Oral Health; Temporomandibular Joint Disorder; Xerostomia.

This is an Open Access article distributed under the terms of the Creative Commons Attribution Non-Commercial License (http://creativecommons.org/licenses/by-nc/4.0/) which permits unrestricted non-commercial use, distribution, and reproduction in any medium, provided the original work is properly cited.

Fibromyalgia (FM) is a chronic pain syndrome characterized by widespread musculoskeletal pain and specific sites of tenderness, stiffness, and fatigue. In particular, specific tender regions are detected without signs of inflammation in patients with FM. This can severely decrease the quality of life and affect any region in a patient, including the orofacial area $[1,2]$. Oral care practitioners can detect the early symptoms of FM and assist patients in receiving the proper diagnosis and treatment. The epidemiology, pathophysiology, clinical manifestation, diagnosis, orofacial concerns, and treatment of FM are reviewed here.

\section{EPIDEMIOLOGY}

FM has been diagnosed in $3-6 \%$ of the population, imposing substantial medical costs [3]. It occurs in up to $4 \%$ of men and $2.5-10.5 \%$ of women with a peak onset of disease in middle age (45-60 years). FM has a familial preponderance with variable manifestations [4,5]. It is prevalent in lower socioeconomic groups, which have members more likely to work in manual occupations, leading to an increase in trauma and pain [6,7].

\section{PATHOPHYSIOLOGY}

The etiology of FM is unknown. It is associated with a multifactorial etiology. First-degree relatives with FM are 8.5 times more likely to have this disorder than the general population [5]. However, genetic factors associated with FM are unknown. It has been suggested that serotonin- and dopamine-related genes may play a role in the pathogenesis of FM [8].

Many researchers report that patients with FM have

Received: August 29, 2020 - Revised: October 4, 2020 - Accepted: October 5, 2020

Corresponding Author: Younghoon Jeon, Department of Anesthesiology and Pain Medicine, School of Dentistry, Kyungpook National University, 130 Dongdeok-ro, Jung-gu, Daegu 41944, Republic of Korea

Tel: +82-53-200-5871 Fax: +82-53-426-2760 E-mail: jeon68@gmail.com

Copyright(c) 2020 Journal of Dental Anesthesia and Pain Medicine 
neuroendocrine dysfunctions such as hyperactivity of the hypothalamic-pituitary-adrenal (HPA) axis and sympathoadrenal system, and relative hypocortisolism $[9,10]$. Emotional and physical stress can affect the HPA axis $[9,10]$.

Patients with FM have a three times higher concentration of substance $\mathrm{P}$ in the cerebrospinal fluid than healthy controls [11]. Activation of the N-methyld-aspartate receptor (NMDAR) is increased in FM patients. Substance $\mathrm{P}$ modulates the responsiveness of the NMDAR to glutamate, which consequently leads to temporary central sensitization and temporal summation in otherwise healthy individuals $[12,13]$. It has been demonstrated that serotonin levels in the serum are reduced and inversely correlated with pain threshold in FM patients [14,15]. Combined dysfunctional neurotransmitter systems, such as low serotonin and high substance $\mathrm{P}$ levels, can produce more pain than either abnormality on their own and be responsible for the onset of FM [16,17].

Dysfunction of the autonomic nervous system is common in FM patients. Positional changes often result in syncope, palpitations, and dizziness [18,19]. Heart rate variability among FM patients is reduced due to an increased nocturnal exaggerated sympathetic modulation of the sinus node $[18,19]$. Abnormal functioning of the HPA axis leads to dysregulation of diurnal cortisol production, which can result in cortisol deficiency and maladaptation [14]. This abnormal chronobiology is linked to sleep disturbances and fatigue in FM patients [14,19].

Research has demonstrated that FM patients have a lower level of dopamine, which plays a central role in modulating pain perception and natural analgesia within supraspinal regions and the spine in painful conditions $[20,21]$. It was found that several serum pro-inflammatory cytokines, such as tumor necrosis factor (TNF)- $\alpha$ and interleukin, are involved in the generation of symptoms in FM, including sleep disturbances, fatigue, and myalgia [8,22-24]. It has been suggested that oxidative stress may also play a role in the pathophysiology of FM. Several studies have shown that lipid peroxidation is correlated with pain, depression, and quality of life in FM patients $[24,25]$. The severity of pain was significantly associated with oxidized low-density lipoprotein [24].

Physical trauma, infection, and psychosocial factors are reported to be associated with the onset of FM [26,27]. Environmental triggers, such as acute illness and psychosocial stress, may alter the pain modulatory response in the brain, leading to enhanced pain perception [27].

\section{CLINICAL MANIFESTATIONS AND DIAGNOSIS}

FM is a complex systemic disorder characterized by generalized musculoskeletal pain and specific sites of tenderness, sleep disturbance, stiffness and fatigue, and psychological problems [1,2]. The diagnosis of fibromyalgia is based predominantly on the patient's history and physical examination findings. However, it is difficult because patients with FM may have several comorbid conditions, such as a history of headaches, temporomandibular disorder (TMD), irritable bowel syndrome, interstitial cystitis, myofascial pain syndrome, and restless leg syndrome [1,28]. FM is defined as a chronic musculoskeletal pain syndrome of unknown etiology, characterized by widespread pain for more than 3 months and tenderness in at least 11 out of 18 tender point sites by the American College of Rheumatology (Table 1) [29].

Psychiatric conditions, such as depression, panic disorder, anxiety, and post-traumatic stress disorder, are common in FM patients [30]. FM patients are more than three times as likely to have psychiatric disorders than the general population. It was found that the psychiatric disorders associated with FM greatly compromise the quality of life of affected patients [31,32]. Sleep disturbances such as non-restorative sleep, insomnia, and poor quality sleep, are reported to be higher in patients with FM, which is strongly associated with pain and fatigue $[1,33]$. 
Table 1. Excluded studies with reasons

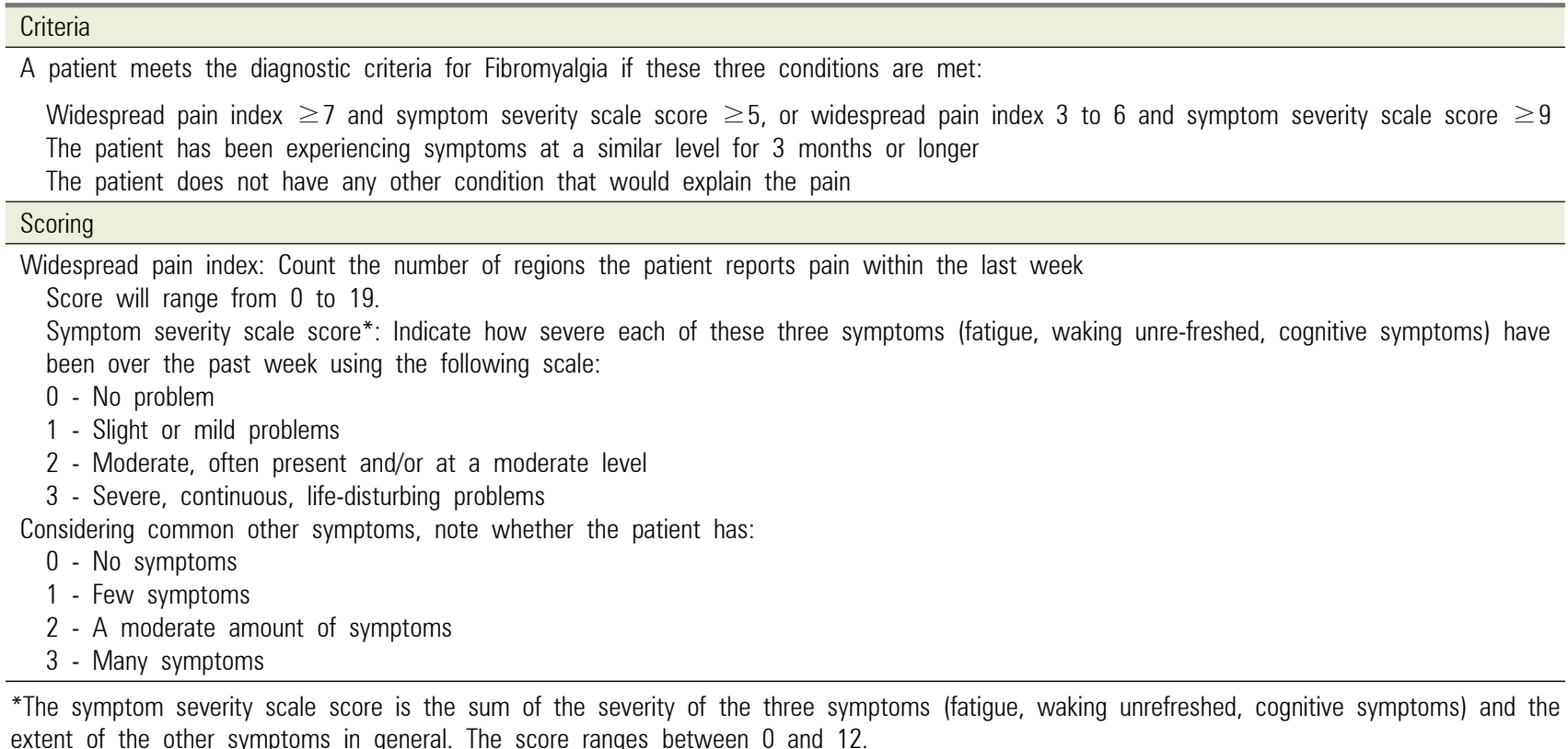

\section{OROFACIAL CONCERNS ASSOCIATED WITH FM}

Many patients with FM have orofacial pain disorders, such as TMD and oral complaints [22,34].

TMD is a term encompassing chronic orofacial pain conditions involving the masticatory muscles, temporomandibular joint, and associated structures. The exact etiology and progression of TMD are poorly understood, while the primary pathology appears to be a degenerative condition, such as osteoarthritis or osteoarthrosis [22,35]. The prevalence of TMD ranges from $42 \%$ to $94 \%$ in FM patients $[35,36]$. Therefore, it was suggested that FM may be a predisposing factor for the onset of TMD [37,38]. It has been reported that the masticatory muscles are sensitive and can be tender points in patients with FM [37]. The comorbidity of TMD and FM may lead to or be a consequence of the centrally mediated alteration in pain perception [39-41]. Therefore, routine treatments for patients with TMD and FM may not be effective [39]. Occlusal splints were found to be ineffective in relieving myofascial pain in patients with widespread pain [42]. It was reported that full body tactile stimulation using massage was effective to improve the signs and symptoms of TMD in patients who are refractory to conservative TMD treatment [43]. Failure to diagnose underlying FM may not provide appropriate treatment. FM patients with TMD are associated with several comorbidities and psychosocial problems.

FM patients may present with numerous oral complaints, such as xerostomia, glossodynia, and dysgeusia.

Xerostomia, known as dry mouth, is a subjective sensation of dryness in the mouth, which often accompanies salivary gland hypofunction. The incidence of xerostomia with FM varies from $7 \%$ to $71 \%[39,44]$. FM patients with various comorbidities are usually managed with xerostomia-inducing medications including antidepressants, sedatives, and muscle relaxants [44,45]. It was demonstrated that about $71 \%$ of FM patients have xerostomia, while only $27.5 \%$ of FM patients receive xerogenic medications [44]. This suggests a significant prevalence of xerostomia in FM patients independent of the use of xerogenic medications [44]. Xerostomia can increase the rates of caries, periodontal disease, dysphasia, mouth ulcers, and candidiasis. Therefore, it is important to provide appropriate treatment for xerostomia 
and avoid oral complications [45].

Glossodynia (burning mouth syndrome) is also commonly noted in FM patients. Approximately 33\% of patients with FM experience glossodynia [44]. Hormonal disturbance, malnutrition, and depression are also associated with burning mouth syndrome [44,46]. The exact etiology and pathogenesis of burning mouth syndrome remains unclear. Neurological mechanisms, including peripheral neurogenic damage and central hyperexcitability, may induce hyperalgesia and allodynia in FM patients with glossodynia [39,44]. Antidepressants, such as amitriptyline, are reported to be effective in treating FM with glossodynia [39,47]. In addition, the use of saliva substitutes and sialagogues and avoiding consumption of alcohol and caffeine are recommended for FM patients with xerostomia [39,47].

Dysgeusia is a taste disorder that causes foul, rancid, metallic, or salty taste perception. Dysgeusia was reported in $34.2 \%$ of FM patients [44]. This can represent a symptom of somatization or side effect of xerogenic medications $[44,46]$.

Headache is reported by $35 \%$ to $82 \%$ of FM patients $[48,49]$. Migraine or tension-type headache is common in FM patients. It was demonstrated that chronic headache was endorsed by $76 \%$ of treatment-seeking FM patients, with $84 \%$ reporting substantial or severe impact from their headaches $[40,48]$. Therefore, it was suggested that assessment of headache should be a part of the routine evaluation of patients with FM [49].

\section{PATIENT MANAGEMENT}

The clinical presentation of FM varies. Patients should be questioned about a detailed history of orofacial complaints, current medications, and commodities. When the diagnosis of FM is suspected, the patients can be referred to a pain physician or rheumatologist for appropriate diagnostic workup and treatment [46].

A multidisciplinary approach is required for the treatment of patients with FM. Education, pharmaco-
Table 2. Treatments for fibromyalgia

\begin{tabular}{l}
\hline Education \\
\hline Nature of fibromyalgia \\
The difference between the chronic, widespread pain and pain from \\
an oral disease or infection \\
\hline Pharmacologic therapy \\
\hline Tricyclic antidepressants: amitriptyline, nortriptyline \\
Selective serotonin reuptake inhibitors: fluoxetine, sertraline, \\
paroxetine \\
Serotonin-norepinephrine reuptake inhibitors: venlafaxine, duloxetine \\
Muscle relaxants: cyclobenzaprine, tizanidine \\
Anticonvulsants: pregabalin \\
Sedative hypnotics: zolpidem \\
Analgesics: tramadol, opioid, nonsteroidal anti-inflammatory drugs \\
\hline Nonpharmacologic therapy \\
Cognitive behavioral therapy \\
Relaxation therapy \\
Low impact and aerobic exercises \\
Soft tissue massage \\
Medicinal bath \\
Trigger point and tender point injections \\
Combined therapy of ultrasound and inferential current
\end{tabular}

logical therapy, and nonpharmacological therapy are the mainstay of treating pain and associated conditions in patients with FM (Table 2). [1,2]. Strategies to reduce stress during oral care, build a trusting relationship between the patient and the practitioner, and provide effective pain management are required [39]. One of the most important aspects in the treatment of FM is to recognize the nature of this disease. FM is not an acute but chronic condition $[39,46,50]$. Therefore, the aim of treatment is to manage symptoms of FM, such as pain, sleep disturbance, and depression. A variety of medications are used to treat the symptoms of FM (Table 2). Polypharmacy is usually employed. Pharmacological treatment should be guided toward pain as well as comorbidities. Healthcare providers should be familiar with the side effects of drugs and possible adverse drug reactions associated with multiple medications. For instance, combined treatment with antidepressants and anticonvulsants can increase dry mouth, sedation, dizziness, and constipation, compared with that with each treatment alone [50].

Pharmacological treatment alone is often ineffective in treating FM [39]. The combination of education, cognitive behavioral therapy, and exercise regimens with 
pharmacologic treatment are important for managing FM [50]. These therapies are reported to provide long-term beneficial effects with an improvement in symptoms and overall quality of life $[46,49,50]$. Psychological interventions, including basic body awareness therapy, cognitive-behavioral therapy, and imagery intervention, are often used for FM [39,50]. Other nonpharmacologic therapies such as heat application and dietary modulation are also effective [50].

Due to pain and comorbidities, it is often difficult for patients with FM to perform effective oral health self-care, which can lead to perioral lesions such as ulceration and aphthous stomatitis [36]. Perioral infection exacerbates stress on the body, which consequently worsens the symptoms of FM. Oral health care providers should frequently monitor oral self-care and help prevent oral diseases and infections $[39,46]$.

FM patients commonly have various psychiatric disorders. It is important to reduce emotional stress and anxiety prior to their oral hygiene treatment. In addition, FM patients often complain of heightened pain sensitivity and fatigue [27]. Poor management of pain and stress can lead to adverse patient outcomes. Therefore, pretreatment with anti-anxiety drugs and topical and local anesthetic agents is beneficial for the management of discomfort during oral healthcare $[39,50]$.

\section{CONCLUSION}

FM affects the overall health of patients, and FM patients commonly present with a variety of oral manifestations, including temporomandibular disorder, xerostomia, glossodynia, and dysgeusia. Therefore, oral healthcare providers need to be aware of this disorder to better manage oral symptoms and provide proper oral self-care modification and education about the nature of FM. Additionally, it should be considered to provide a stress-free treatment environment.
AUHOR OROGDS

Younghoon Jeon: https://orcid.org/0000-0002-7168-4214

\section{AUHOR GONIRIBUIOIS}

Younghoon Jeon: Writing, review, \& editing

NOTE: No conflicts of interest or funding.

\section{REFERENCES}

1. Clauw DJ. Fibromyalgia: a clinical review. JAMA 2014; 311: $1547-55$.

2. Morin AK. Fibromyalgia: a review of management options. Formulary 2009; 44: 362-73.

3. Goldenberg DL, Simms RW, Geiger A, Komaroff AL. High frequency of fibromyalgia in patients with chronic fatigue seen in a primary care practice. Arthritis Rheum 1990; 33: 381-7.

4. Neumeister MW, L Neumeister EL. Fibromyalgia. Clin Plastic Surg 2020; 47: 203-13.

5. Arnold LM, Hudson JI, Hess EV, Ware AE, Fritz DA, Auchenbach MB, et al. Family study of fibromyalgia. Arthritis Rheum 2004; 50: 944-52.

6. Bergman S, Herrström P, Högström K, Petersson IF, Svensson B, Jacobsson LT. Chronic musculoskeletal pain, prevalence rates, and sociodemographic associations in Swedish population study. J Rheumatol 2001; 28: 1369-77.

7. Bergman S. Psychosocial aspects of chronic widespread pain and fibromyalgia. Disabil Rehabil 2005; 27: 675-83.

8. Buskila D, Neumann L, Press J. Genetic factors in neuromuscular pain. CNS Spectr 2005; 10: 281-4.

9. Adler GK, Kinsley BT, Hurwitz S, Mossey CJ, Goldenberg DL. Reduced hypothalamic-pituitary and sympathoadrenal responses to hypoglycemia in women with fibromyalgia syndrome. Am J Med 1999; 106: 534-43.

10. Heim C, Ehlert U, Hellhammer DH. The potential role of hypocortisolism in the pathophysiology of stress-related bodily disorders. Psychoneuroendocrinology 2000; 25: $1-35$. 
11. Russell IJ, Orr MD, Littman B, Vipraio GA, Alboukrek D, Michalek JE, et al. Elevated cerebrospinal fluid levels of substance $\mathrm{P}$ in patients with the fibromyalgia syndrome. Arthritis Rheum 1994; 37: 1593-601.

12. Pyke T, Osmotherly PG, Baines S. Measuring glutamate levels in the brains of fibromyalgia patients and a potential role for glutamate in the pathophysiology of fibromyalgia symptoms: a systematic review. Clin J Pain 2017; 33: 94454.

13. Dhar M. Pathophysiology and clinical spectrum of fibromyalgia: a brief overview for medical communicators. AMWA Journal 2011; 26: 50-4.

14. Wolfe F, Russell IJ, Vipraio G, Ross K, Anderson J. Serotonin levels, pain threshold, and fibromyalgia symptoms in the general population. J Rheumatol 1997; 24: $555-9$.

15. Ernberg M, Voog U, Alstergren P, Lundeberg T, Kopp S. Plasma and serum serotonin levels and their relationship to orofacial pain and anxiety in fibromyalgia. J Orofac Pain 2000; 14: 37-46.

16. Cordero MD, Alcocer-Gómez E, Cano-García FJ, de Miguel M, Sánchez-Alcázar JA, Moreno Fernández AMM. Low levels of serotonin in serum correlates with severity of fibromyalgia. Med Clin (Barc) 2010; 13; 135: 644-6.

17. Becker S, Schweinhardt P. Dysfunctional neurotransmitter systems in fibromyalgia, their role in central stress circuitry and pharmacological actions on these systems. Pain Res Treat 2012; 2012: 741-6.

18. Staud R. Autonomic dysfunction in fibromyalgia syndrome: postural orthostatic tachycardia. Curr Rheumatol Rep 2008; 10: 463-6.

19. Martínez-Lavín M, Hermosillo AG, Rosas M, Soto ME. Circadian studies of autonomic nervous balance in patients with fibromyalgia: a heart rate variability analysis. Arthritis Rheum 1998; 41: 1966-71.

20. Wood PB, Schweinhardt P, Jaeger E, Dagher A, Hakyemez $\mathrm{H}$, Rabiner EA, et al. Fibromyalgia patients show an abnormal dopamine response to pain. Eur J Neurosci 2007; 25: $3576-82$.

21. Wood PB. Role of central dopamine in pain and analgesia. Expert Rev Neurother 2008; 8: 781-97.

22. Kutu FC, Özdolap Ş, Sarikaya S. Pro-inflammatory cytokines and oxidized low-density-lipoprotein in patients with fibromyalgia. Arch Rheumatol 2018; 34: 123-9.

23. Lucas HJ, Brauch CM, Settas L, Theoharides TC. Fibromyalgia--new concepts of pathogenesis and treatment. Int J Immunopathol Pharmacol 2006; 19: 5-10.

24. Ranzolin A, Duarte AL, Bredemeier M, da Costa Neto CA, Ascoli BM, Wollenhaupt-Aguiar B, et al. Evaluation of cytokines, oxidative stress markers and brain-derived neurotrophic factor in patients with fibromyalgia - a controlled cross-sectional study. Cytokine 2016; 84: 25-8.

25. La Rubia M, Rus A, Molina F, Del Moral ML. Is fibromyalgia-related oxidative stress implicated in the decline of physical and mental health status? Clin Exp Rheumatol 2013; 31: 121-7.

26. Jiao J, Vincent A, Cha SS, Luedtke CA, Kim CH, Oh TH. Physical trauma and infection as precipitating factors in patients with fibromyalgia. Am J Phys Med Rehabil 2015; 94: 1075-82.

27. Adams LM, Turk DC. Psychosocial factors and central sensitivity syndromes. Curr Rheumatol Rev 2015; 11: 96-108.

28. Hedenberg-Magnusson B, Ernberg M, Kopp S. Symptoms and signs of temporomandibular disorders in patients with fibromyalgia and local myalgia of the temporomandibular system. A comparative study. Acta Odontol Scand 1997; 55: 344-9.

29. Wolfe F, Clauw DJ, Fitzcharles MA, Goldenberg DL, Katz RS, Mease P, et al. The american college of rheumatology preliminary diagnostic criteria for fibromyalgia and measurement of symptom severity. Arthritis Care Res 2010; 62: $600-10$

30. Buskila D, Cohen H. Comorbidity of fibromyalgia and psychiatric disorders. Curr Pain Headache Rep 2007; 11: 333-8.

31. Burke NN, Finn DP, Mcguire BE, Roche M. Psychological stress in early life as a predisposing factor for the development of chronic pain: clinical and preclinical evidence and neurobiological mechanisms. J Neurosci Res 2017; 95: 1257-70

32. Carta MG, Moro MF, Pinna FL, Testa G, Cacace E, Ruggiero V, et al. The impact of fibromyalgia syndrome 
and the role of comorbidity with mood and post-traumatic stress disorder in worsening the quality of life. Int J Soc Psychiatry 2018; 64: 647-55.

33. Munguía-Izquierdo D, Legaz-Arrese A. Determinants of sleep quality in middle-aged women with fibromyalgia syndrome. J Sleep Res 2012; 21: 73-9.

34. Pimentel MJ, Gui MS, de Aquino LM, Rizzatti-Barbosa CM. Features of temporomandibular disorders in fibromyalgia syndrome. Cranio 2013; 31: 40-5.

35. Hedenberg-Magnusson B, Ernberg M, Kopp S. Presence of orofacial pain and temporomandibular disorder in fibromyalgia. A study by questionnaire. Swed Dent J 1999; 23: $185-92$.

36. Korszun A, Papadopoulos E, Demitrack M, Engleberg C, Crofford L. The relationship between temporomandibular disorders and stress-associated syndromes. Oral Surg Oral Med Oral Pathol Oral Radiol Endod 1988; 86: $416-20$.

37. Leblebici B, Pektaş ZO, Ortancil O, Hürcan EC, Bagis $\mathrm{S}$, Akman MN. Coexistence of fibromyalgia, temporomandibular disorder, and masticatory myofascial pain syndromes. Rheumatol Int 2007; 27: 541-4.

38. Velly AM, Look JO, Schiffman E, Lenton PA, Kang W, Messner RP, et al. The effect of fibromyalgia and widespread pain on the clinically significant temporomandibular muscle and joint pain disorders--a prospective 18-month cohort study. J Pain 2010; 11: 1155-64.

39. Walters A, Tolle SL, McCombs GM. Fibromyalgia syndrome: considerations for dental hygienists. J Dent Hyg 2015; 89: 76-85.

40. Aaron LA, Buchwald D. Chronic diffuse musculoskeletal pain, fibromyalgia and co-morbid unexplained clinical conditions. Best Pract Res Clin Rheumatol 2003; 17: 563-74.

41. Nguyen TT, Vanichanon P, Bhalang K, Vongthongsri S.
Pain duration and intensity are related to coexisting pain and comorbidities present in temporomandibular disorder pain patients. J Oral Facial Pain Headache 2019; 33: 205-12. 42. Raphael KG, Marbach JJ. Widespread pain and the effectiveness of oral splints in myofascial face pain. J Am Dent Assoc 2001; 132: 305-16.

43. Adiels AM, Helkimo M, Magnusson T. Tactile stimulation as a complementary treatment of temporomandibular disorders in patients with fibromyalgia syndrome. A pilot study. Swed Dent J 2005; 29: 17-25.

44. Rhodus NL, Fricton J, Carlson P, Messner R. Oral symptoms associated with fibromyalgia syndrome. J Rheumatol 2003; 30: 1841-45.

45. Guggenheimer J, Moore PA. Xerostomia: etiology, recognition and treatment. J Am Dent Assoc 2003; 134: 61-9.

46. Balasubramaniam R, Laudenbach JM, Stoopler ET. Fibromyalgia: an update for oral health care providers. Oral Surg Oral Med Oral Pathol Oral Radiol Endod 2007; 104: 589-602.

47. Peterson EL. Fibromyalgia: management of a misunderstood disorder. J Am Acad Nurse Pract 2007; 19: 341-8.

48. Okifuji A, Turk DC, Marcus DA. Comparison of generalized and localized hyperalgesia in patients with recurrent headache and fibromyalgia. Psychosom Med 1999; 61: 771-80.

49. Marcus DA, Bernstein C, Rudy TE. Fibromyalgia and headache: an epidemiological study supporting migraine as part of the fibromyalgia syndrome. Clin Rheumatol 2005; 24: 595-601.

50. Macfarlane GJ, Kronisch C, Dean LE, Atzeni F, Häuser W, Fluß E, et al. EULAR revised recommendations for the management of fibromyalgia. Ann Rheum Dis 2017; 76: 318-28. 